\title{
Numerical Analysis of Pattern Shape Deformation in UV Imprint Due to Curing Shrinkage
}

\author{
Yuki Onishi $^{1^{*}}$, Ryunosuke Yamashita ${ }^{1}, K_{\text {Kenji Amaya }}{ }^{1}$, and Yoshihiko Hirai ${ }^{2}$ \\ ${ }^{1}$ Department of Systems and Control Engineering, School of Engineering, \\ Tokyo Institute of Technology, 2-12-1 O-okayama, Meguro-ku, Tokyo 152-8550, Japan \\ ${ }^{2}$ Department of Physics and Electronics, Graduate School of Engineering, \\ Osaka Prefecture University, 1-1 Gakuen-cho, Naka-ku, Sakai, Osaka 599-8531, Japan \\ *yonishi@a.sc.e.titech.ac.jp
}

\begin{abstract}
The accuracy of a numerical modeling method for UV imprint processes to simulate the UV resin deformation due to UV shrinkage was evaluated. Typical UV resins induce volumetric shrinkage by several percent after UV curing. The curing shrinkage deforms the pattern shape and could be a significant issue in UV imprint for high-precision pattern moldings such as optical device patterning. Taking notice of the analogy between UV imprint and thermal imprint, the modeling method introduces the idea of "virtual temperature" to express the UV reaction progress. Accordingly, the curing/shrinkage of UV resin can be treated as the solidification /contraction of thermo-plastic resin modeled as a thermoviscoelastic material. A validation analysis for a micromirror array patterning with the shift in the demolding time reveals that the modeling method can qualitatively predict the final shape of surface curves varied with the demolding time.

Keywords: UV imprint, UV curing, UV shrinkage, Thermo-viscoelastic material, Virtual temperature, Finite element analysis
\end{abstract}

\section{Introduction}

UV imprinting is known as a low-cost and high throughput manufacturing method for a wide range of microstructures. In recent years, the use of UV imprinting has been considered in the production of microscopic optical devices such as microlens arrays. However, UV resin is known to cause several percent volume shrinkage upon curing [14]. When a soft mold such as polydimethylsiloxane (PDMS) is used, the mold is deformed by the UV shrinkage of the UV resin, and the deformation is directly transferred to the surface of the UV resin (see Fig. 1). This transfer error can result in an unexpected curve on the pattern surfaces. In the case of high-precision optical devices, such a surface curve could be a significant issue. Therefore, numerical methods to predict the pattern shape deformation of the UV resin due to curing shrinkage in advance is necessary to be developed.

There are several prior researches on mechanical simulation on UV imprint [5-10]; however, none of them considered the time-dependent curing and shrinkage of the UV resin simultaneously.
Meanwhile, in the area of thermal imprint, there are some prior numerical researches [11-17], and several of them consider both the time-dependent deformation and thermal contraction of the thermoplastic resin. Hirai et al. [15] proposed a numerical method for the thermal imprint process using a hyperelastic material model in the framework of solid mechanics. As an extension of Hirai's approach, Onishi and Takagi et al. [16,17] showed the effectiveness of a thermoviscoelastic model in thermal imprint process simulation. In

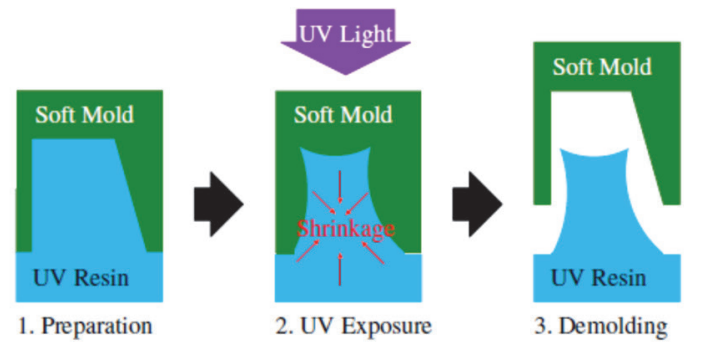

Fig. 1. Mechanism of pattern shape deformation in UV imprint due to UV shrinkage. 
these methods, the time-dependent deformation of the resin is reproduced by using the generalized Maxwell model and the time-temperature superposition principle. Because UV curing/shrinkage is a complex physical phenomenon with a phase change from a fluid phase to a solid phase, the numerical modeling of UV imprint is considered to be more complicated than that of thermal imprint.

Recently, our group proposed a time-dependent numerical modeling method for the UV imprint process, taking into account the curing/shrinkage of the UV resin and the deformation of the soft mold [18]. In the modeling method, the UV curing/shrinkage phenomena of the UV resin was replaced by the cooling solidification/contraction phenomena of the thermo-viscoelastic material, respectively, by introducing the idea of "virtual temperature". The phase change of the UV resin was not strictly handled but was approximately modeled as a viscoelastic solid with a sufficiently small longterm shear modulus. By considering the contact between the UV resin and the mold, the curve on the UV resin surface after demolding was predicted using 3D finite element analysis. However, the validity of the modeling method in various test conditions has not been studied yet. The change of the final surface curve due to the shift in the demolding time is an issue especially important to manufacturing throughput.

In this study, we examine the effect of demolding time on the final pattern shape deformation from both experimental and numerical analyses and evaluate the validity of the numerical analysis based on our modeling method [18]. Using a micromirror array as an example, we show the final shapes of the top surface tested with two different demolding time cases, and also show the results of numerical analysis under the same conditions to discuss the validity of the numerical method.

\section{Methods}

This section briefs our modeling method [18]. Note that some experimental data were re-acquired for this study and are not identical to those in the reference.

\subsection{Outline}

UV resin is a fluid before UV curing and changes phase to a solid after UV curing. First of all, it is necessary to consider whether to adopt the fluid dynamics approach or the solid mechanics approach in the UV imprint process simulation. In this study, we mainly focus on the shrinkage of the UV resin and the deformation of the soft mold, as shown in Fig. 1. In addition, the flow of UV resin in the fluid phase after UV exposure is relatively small because the UV curing usually arises shortly after the UV exposure [18]. Therefore, it is considered that the solid mechanics approach is more appropriate when dealing with the deformations targeted in this study. The time history of the stiffness of UV resin after UV exposure usually shows a curve similar to thermo-plastic resin under cooling solidification [18]. Also, the time history of the volume change of UV resin after UV exposure is similar to that of thermo-plastic resin under cooling contraction. Focusing on these analogies and referring to the methods for thermal imprint process simulation [16, 17], the proposed method uses a thermoviscoelastic constitutive model to represent the mechanical behavior of the UV resin.

By introducing "virtual temperature", various phenomena of UV resin can be treated as those of thermo-plastic resin as follows.

\section{Progress of UV reaction \\ $\Rightarrow$ Decrease in virtual temperature}

Monotonical decrease in virtual temperature of the resin simulates the progress of UV reaction.

\section{UV curing $\Rightarrow$ Cooling solidification}

Cooling solidification of the thermoviscoelastic body simulates the stiffening of the UV resin.

\section{UV shrinkage $\Rightarrow$ Cooling contraction}

Thermal contraction of the thermoviscoelastic body simulates the shrinkage of the UV resin.

The UV resin focused on this research is a cation polymerization-type UV resin (Daicel Co., Tokyo, Japan [19]). Note that the UV curing and shrinkage continue even after the UV exposure because of the dark curing (a.k.a. shadow cure or post-cure effect).

\subsection{Mathematical formulation}

\subsubsection{Virtual temperature: $\theta^{\text {virt }}(t)$}

In this study, we focus on only one UV exposure condition $\left(365 \mathrm{~nm}\right.$, uniform $50 \mathrm{~mW} / \mathrm{cm}^{2}$ in intensity from $t=0$ till $30 \mathrm{~s}$ ) for simplicity. Then, the time history of the virtual temperature of the UV resin $\left(\theta^{\text {virt }}(t)\right)$ is set as

$$
\theta^{\mathrm{virt}}(t)=-t \text {, }
$$


where $t$ denotes the time from the start of UV exposure. Note that $\theta^{\text {virt }}$ is just a virtual parameter and has no relation to the real temperature. In order to prevent unnecessary confusion, we omit the unit assignment to $\theta^{\text {virt }}$ in this study.

\subsubsection{Expansion coefficient: $\alpha\left(\theta^{\text {virt }}\right)$}

Figure 2 shows an experimental result with a rotational oscillating rheometer (MCR301, Anton Paar, Graz, Austria [20]) measuring the thicknesstime history of UV resin during and after UV exposure. The relative thickness change as a function of time $(\beta(t))$ is defined as

$$
\beta(t)=\frac{(\text { Thickness at } t)-(\text { Initial thickness })}{\text { (Initial thickness) }}
$$

Assuming $\theta^{\text {virt }}(0)=0$, the linear thermal expansion coefficient as a function of virtual temperature $\left(\alpha\left(\theta^{\text {virt }}\right)\right)$ is written as

$$
\alpha\left(\theta^{\mathrm{virt}}\right)=\frac{1}{3} \beta(-t)
$$

Smoothing the experiment data appropriately, the curve of $\alpha\left(\theta^{\text {virt }}\right)$ is obtained as shown in Fig. 3.

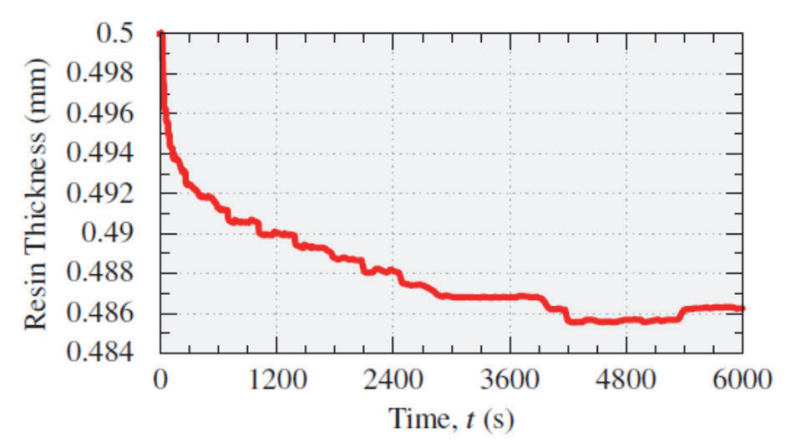

Fig. 2. Thickness-time history of the UV resin during and after the UV exposure on a rheometry test.

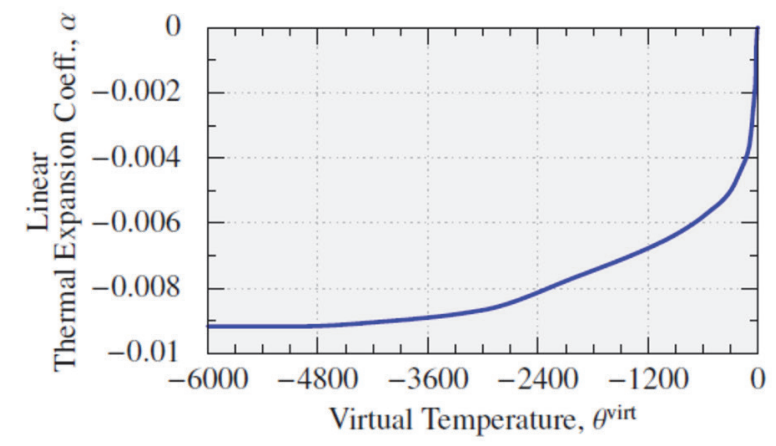

Fig. 3. Identified linear thermal expansion coefficient as a function of virtual temperature.

\subsubsection{Shift factor: $A\left(\theta^{\text {virt }}\right)$}

The material constitutive model of the UV resin in this study is a thermo-viscoelastic material based on the generalized Maxwell model that has a virtual temperature-time shift factor function $\left(A\left(\theta^{\text {virt }}\right)\right)$. The stress/strain formulation of the thermo-viscoelastic materials in a time-dependent analysis is detailed in the references [16,21] and is omitted in this paper. $A\left(\theta^{\text {virt }}\right)$ is identified by the time domain complex shear modulus at various frequencies $G^{*}(t ; f)$. This study deals with $G^{*}(t ; f)$ measured at six different frequencies $(f=10,5,1,0.5,0.1$, and $0.01 \mathrm{~Hz})$ with the rheometer. Then, $G^{*}(t ; f)$ is regarded as $G^{*}\left(-\theta^{\text {virt; }}\right.$ $f$ ) and is converted to the frequency domain function at various virtual temperatures $G^{*}\left(\omega ; \theta^{\text {virt }}\right)$. Shifting $G^{*}\left(\omega ; \theta^{\text {virt }}\right)$ in the frequency coordinate for various $\theta^{\text {virt }}$ appropriately, a smoothed curve at a reference virtual temperature $G^{*}\left(\omega ; \theta_{\mathrm{ref}}^{\text {virt }}\right)$ is obtained as shown in Fig. 4 (note that $G^{*}=G^{\prime}+\mathrm{i} G^{\prime \prime}$, where $G^{\prime}$ is the storage shear modulus and $G$ " is the loss shear modulus). The reference virtual temperature $\left(\theta_{\text {ref }}^{\text {virt }}\right)$ is set to -1800 in this study. Figure 5 shows the factor of the frequency shift as a function of $\theta^{\text {virt }}$, which represents $A\left(\theta^{\text {virt }}\right)$ given by a table data. Note that the data points with $\theta^{\text {virt }}$ less than -1800 are determined by extrapolation.

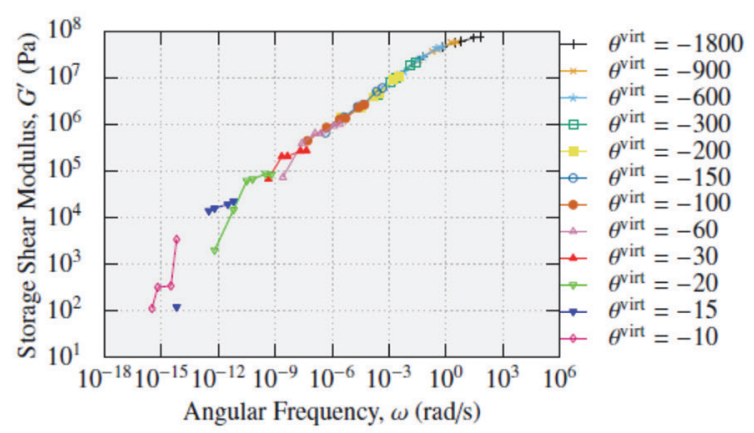

(a) Storage shear modulus.

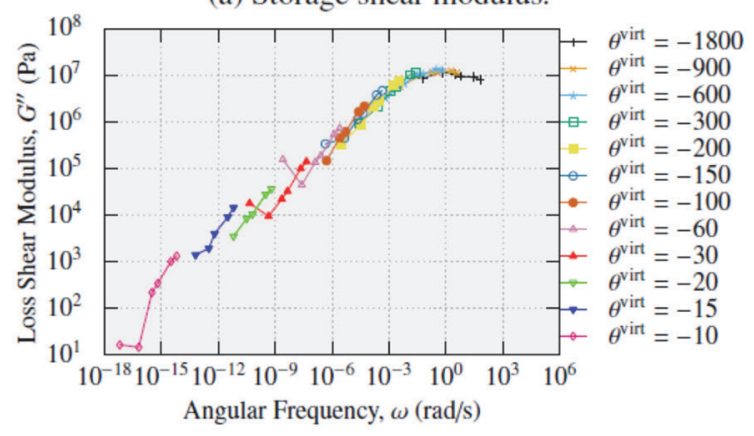

(b) Loss shear modulus.

Fig. 4. Shifted complex shear modulus as a function of angular frequency at the reference temperature. 


\subsubsection{Prony series: $\left(\tau_{i}, g_{i}\right) \mathrm{s}$}

The generalized Maxwell model approximates $G$, and $G$ " as a function of $\omega$ as

$$
\begin{gathered}
G^{\prime}(\omega)=G_{\infty}+G_{0} \sum_{i}\left(g_{i} \frac{\omega^{2} \tau_{i}^{2}}{1+\omega^{2} \tau_{i}^{2}}\right), \\
G^{\prime \prime}(\omega)=G_{0} \sum_{i}\left(g_{i} \frac{\omega \tau_{i}}{1+\omega^{2} \tau_{i}^{2}}\right),
\end{gathered}
$$

where $\left(\tau_{i}, g_{i}\right)$ is the $i$ th term of the Prony series, $G_{0}$ is the instantaneous shear modulus, and $G_{\infty}\left(=G_{0}\right.$ $\left.\left(1-\sum_{i} g_{i}\right)\right)$ is the long-term shear modulus. Since $G_{0}$ can easily be measured by a bulk material test, only $\left(\tau_{i}, g_{i}\right) \mathrm{s}$ are the parameters to be identified. Figure 6 shows the curve of $G^{*}\left(\omega ; \theta_{\text {ref }}^{\text {virt }}\right)$ expressed with the generalized Maxwell model to approximate the experimental data shown in Fig. 4, which is generally called "master curve".

On the other hand, the volumetric modulus is a constant bulk modulus $(K)$ independent of the deformation rate and virtual temperature.

\section{Results and discussion}

In order to confirm the validity of the numerical modeling method, we perform an actual UV imprinting test and a numerical simulation for the patterning of the Parity mirror (Parity Innovations Co., Kyoto, Japan [22]), which is a micromirror array for aerial displays. Figure 7 shows the geometric outline of the analysis. As the analysis model is a 3D model, Fig. 7 shows a cut view on a typical cross-section. In consideration of its repeated structure, the analysis domain is limited to only one pattern by introducing the periodic boundary conditions. The resin is the cation polymerization-type UV resin used in Section 2, whereas the mold is made of PDMS. The UV exposure condition is the same as noted in Section 2, and we assume isotropic curing/shrinkage under the uniform UV intensity distribution. The demolding time is set to $t_{\text {demold }}=130$ and $530 \mathrm{~s}$ to

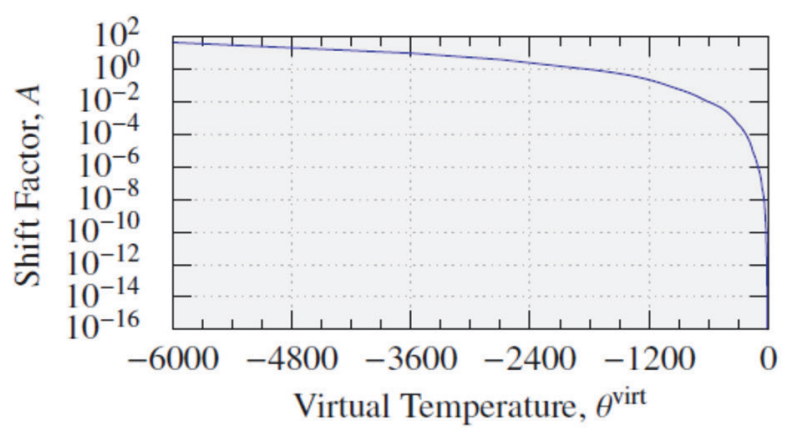

Fig. 5. Shift factor for the complex shear modulus as a function of virtual temperature. evaluate the effect on the surface curve of the top face. The numerical simulation is performed with the general purpose finite element software, ABAQUS/Standard 2017.

Figure 8 compares the surface curve of the top face of the pattern after sufficient time has passed since the demolding. All results show a similar

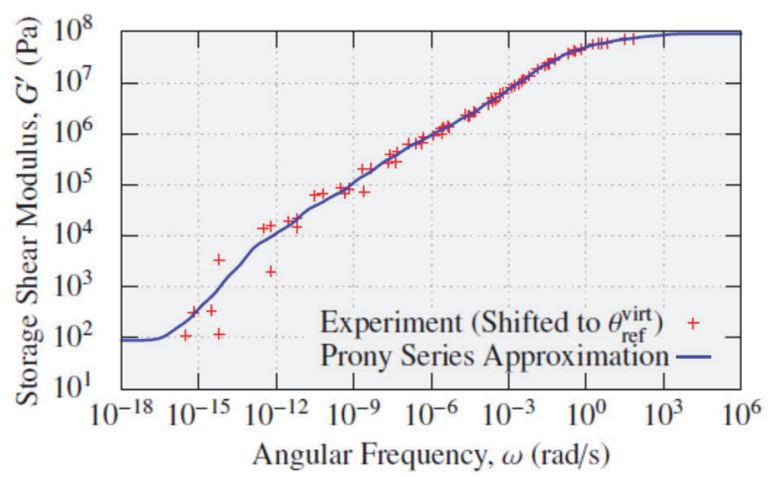

(a) Storage shear modulus.

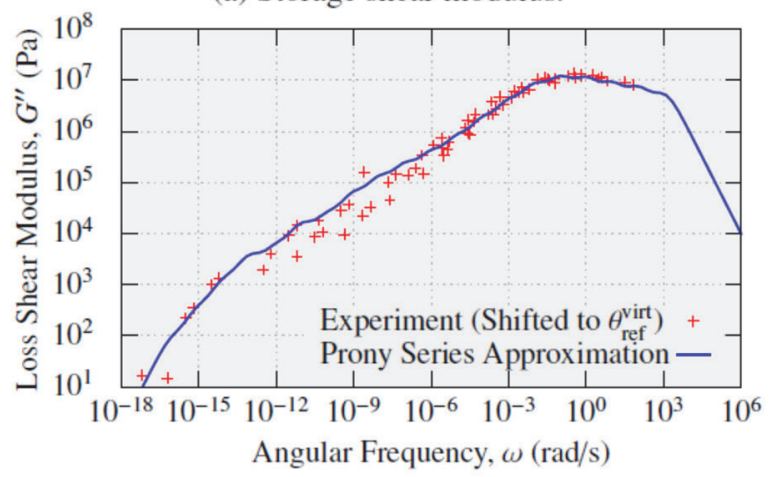

Fig. 6. Prony series approximation of the shifted complex shear modulus as a function of angular frequency at the reference temperature (master curve at $\theta_{\text {ref }}^{\mathrm{virt}}$ ).

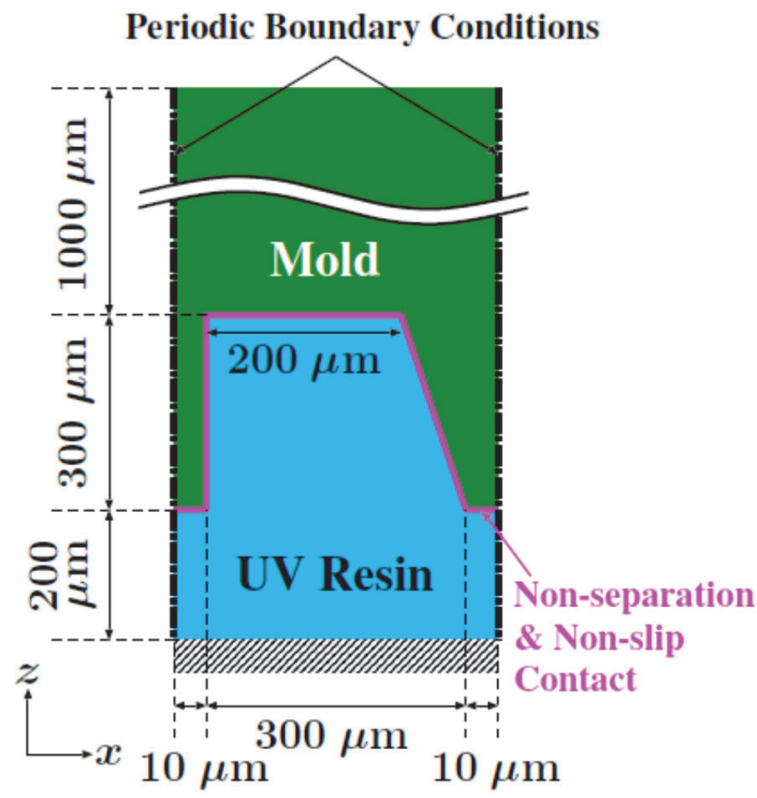

Fig. 7. Geometric outline of the validation analysis (a 2D cross-sectional view). 
Table 1. Comparison of the RMS depth values of the four cases shown in Fig. 8.

\begin{tabular}{ccc}
\hline & $t_{\text {demold }}=130 \mathrm{~s}$ & $t_{\text {demold }}=530 \mathrm{~s}$ \\
Actual & $0.349 \mu \mathrm{m}$ & $0.403 \mu \mathrm{m}$ \\
Numerical & $0.086 \mu \mathrm{m}$ & $0.144 \mu \mathrm{m}$ \\
\hline
\end{tabular}

smooth curve with a concave center. Also, the depth of curve with $t_{\text {demold }}=530 \mathrm{~s}$ is deeper than that with $t_{\text {demold }}=130 \mathrm{~s}$ on both the actual test and numerical simulation. Therefore, this result indicates that the numerical modeling method can predict the surface curve qualitatively. However, the average depth of the curve given by numerical simulation is much smaller than that of the actual imprinting. The error of the RMS depth for each $t_{\text {demold }}=130$ and $530 \mathrm{~s}$ cases is about $-75 \%$ and $-64 \%$, respectively.

It is considered that the consideration of reaction heat generated by the UV reaction could resolve the quantitative error. Our recent experiment shows that the real temperature of the UV resin in the actual imprinting rise to $\sim 140{ }^{\circ} \mathrm{C}$ right after the UV exposure and get back to room temperature gradually. Therefore, the significant decrease in the real temperature after a certain level curing should induce the real thermal contraction in addition to the UV shrinkage (see Fig. 9). Resolving the underestimation issue of the curve depth in numerical simulation is our work in the future.

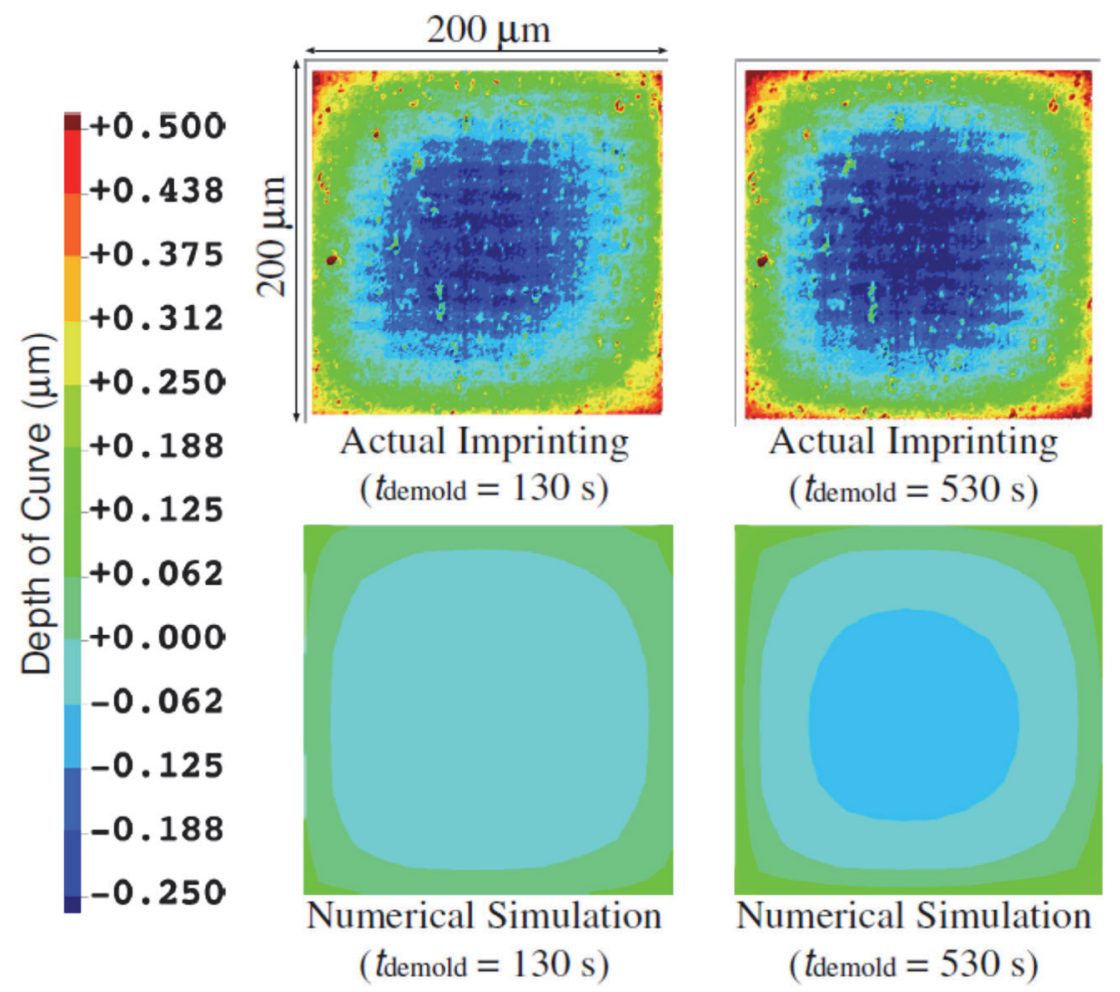

Fig. 8. Comparison of the depth of the curve on the top face of the pattern between the actual imprinting and numerical simulation for two different demolding time cases.

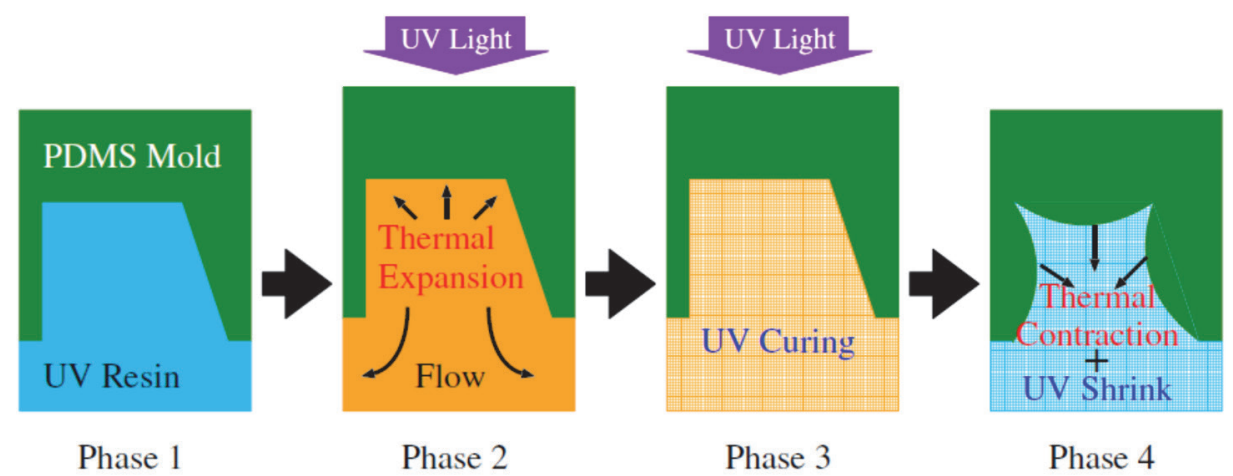

Fig. 9. Mechanism of pattern shape deformation in UV imprint due to both the UV shrinkage and real thermal contraction. 


\section{Conclusion}

The accuracy of the numerical modeling method for UV imprint processes to simulate the UV resin deformation due to UV shrinkage was evaluated. The modeling method introduced the idea of "virtual temperature" to express the UV reaction progress and treated the curing/shrinkage of UV resin as the solidification/contraction of thermoplastic resin. A validation analysis for a micromirror array patterning was conducted and revealed that the proposed method could qualitatively predict the surface shape varied with the shift in the demolding time. Unfortunately, the quantitative validity of the proposed method could not be obtained; yet, consideration of reaction heat generated by the UV reaction has a high possibility of resolving this issue. Further experimental investigation and numerical model improvement are our future work.

\section{References}

1. Q. Wang, H. Hiroshima, and H. Atobe, $J$. Photopolym. Sci. Technol., 23 (2010) 33.

2. R. Suzuki, N. Sakai, A. Sekiguchi, Y. Matsumoto, R. Tanaka, and Y. Hirai, J. Photopolym. Sci. Technol., 23 (2010) 51.

3. H. Hiroshima and K. Suzuki, Jpn. J. Appl. Phys., 50 (2011) 06GK09.

4. H. Min, N. Zheng, Z. Fan, Y. Jiang, and X. Cheng, Microelectron. Eng., 205 (2019) 32.

5. H. Lan, Y. Ding, H. Liu, Y. Que, W. Tao, H. N. Li, and B. Lu, Sci. China Ser. E, 52 (2009) 294.

6. M. Shibata, A. Horiba, Y. Nagaoka, H. Kawata, M. Yasuda, and Y. Hirai, J. Vac. Sci. Technol. B, 28 (2010) 108.

7. A. Amirsadeghi, J. J. Lee, and S. Park, J. Micromech. Microeng., 21 (2011) 115013.
8. A. Horiba, M. Yasuda, H. Kawata, M. Okada, S. Matsui, and Y. Hirai, Jpn. J. Appl. Phys., 51 (2012) 06FJ06.

9. T. Tochino, T. Shiotsu, K. Uemura, M. Yasuda, H. Kawata, and Y. Hirai, J. Vac. Sci. Technol. B, 32 (2014) 06FG08.

10. X. Song, X. Shan, S. Chow, X. Deng, and W. Teo, Microsys. Technol., 21 (2015) 1729.

11. S. Kim, J. Kang, and W. Lee, Polym. Eng. Sci., 51 (2011) 209.

12. W.-B. Young, Microelectron. Eng., 77 (2005) 405.

13. Z. Song, J. Choi, B. H. You, J. Lee, and S. Park, J. Vac. Sci. Technol. B, 26 (2008) 598.

14. H. Hocheng and C. C. Nien, J. Micro/Nanolith. MEMS MOEMS, 5 (2006) 1.

15. Y. Hirai, T. Konishi, T. Yoshikawa, and S. Yoshida, J. Vac. Sci. Technol. B, 22 (2004) 3288.

16. Y. Onishi, Y. Hirai, H. Takagi, M. Takahashi, T. Tanabe, R. Maeda, and Y. Iriye, Jpn. J. Appl. Phys., 47 (2008) 5145.

17. H. Takagi, M. Takahashi, R. Maeda, Y. Onishi, Y. Iriye, T. Iwasaki, and Y. Hirai, Microelectron. Eng., 85 (2008) 902.

18.Y. Onishi, R. Yamashita, K. Amaya, and Y. Hirai, J. Vac. Sci. Technol. B, 37 (2019) 062915.

19. DAICEL Corporation, "DAICEL Co. website", https://www.daicel.com/en/.

20. Anton Paars GmbH, "Anton Paars website", https://www.anton-paar.com/.

21. SIMULIA Inc., SIMULIA ABAQUS 2017 Documentation, SIMULIA Inc. (2017).

22. Parity Innovations Co., "About Parity Innovations", http://www.piq.co.jp/about_e. html. 